\title{
The TOOT Survey: status and early results
}

\author{
Gary J. Hill a Steve Rawlings ${ }^{b}$ \\ ${ }^{a}$ McDonald Observatory, University of Texas at Austin, RLM 15.308, TX 78712, \\ $U S A$ \\ ${ }^{\mathrm{b}}$ Astrophysics, Oxford University, Keble Road, Oxford OX1 3RH, UK
}

\begin{abstract}
The TexOx-1000 (TOOT) radio source redshift survey is designed to find and study typical radio-loud active galaxies to high redshift. They are typical in the same sense that $\mathrm{L}^{*}$ galaxies are typical of galaxies in the optical. Previous surveys have only included the most luminous, rare objects at and beyond the peak of activity at $\mathrm{z} \sim 2$, but in going a factor of 100 fainter than the 3C survey, and in assembling a large sample, TOOT probes for the first time the objects that dominate the radio luminosity density of the universe at high redshift. Here we describe the current status of the TOOT survey and draw preliminary conclusions about the redshift distribution of the radio sources. So far, $\sim 520$ of the 1000 radio sources have redshifts, with $\sim 440$ of those in well-defined, complete, sub-regions of the survey. For these we find a median redshift of $z=1$, but the measured redshift distribution has a deficit of objects with $\mathrm{z} \sim 2$, when compared to predictions based on extrapolating luminosity functions constrained by higher-flux-density samples. These are the more luminous objects that usually show emission lines, and which should not be missed in the survey unless they are heavily reddened. The deficit may be real, but it would not be too surprising to find a population of faint, reddened radio sources at $\mathrm{z} \sim 2-3$ among the TOOT sources yet to have accurate redshifts.
\end{abstract}

Key words: galaxies: active - radio continuum: galaxies - galaxies: surveys: TOOT

\section{Introduction and Motivation}

The TexOx-1000 (TOOT) radio source (RS) survey is an ambitious collaboration between Texas and Oxford Universities to assemble the largest sample of $\mathrm{RS}$, in order to probe typical radio-loud active galaxies to high redshift. The TOOT survey has made use of UK and Texas facilities to identify and measure the redshifts of a large sample drawn from the low-frequency $7 \mathrm{C}$ survey with $\mathrm{S}_{151 \mathrm{MHz}}>0.1 \mathrm{Jy}$. It is already the largest $\mathrm{RS}$ redshift survey in existence that extends to high redshift and is unbiased by optical selection criteria. 


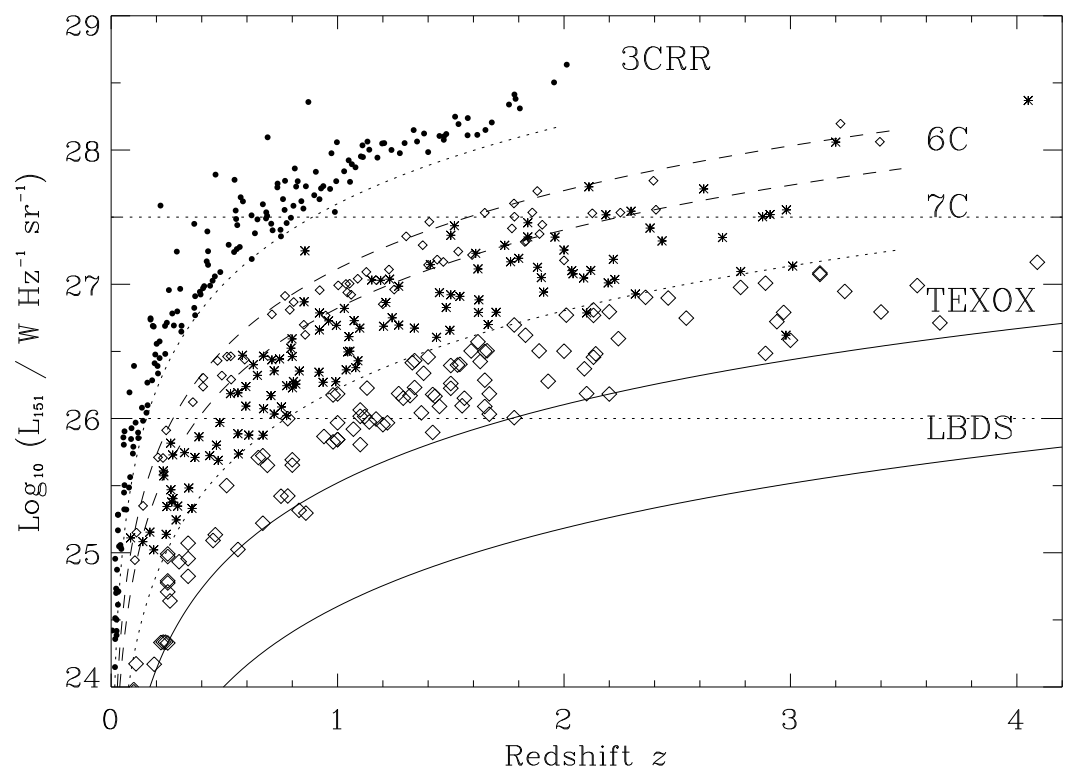

Fig. 1. The Luminosity - Redshift plane for low frequency radio surveys including TOOT. The TOOT survey is labeled as TEXOX. The curved lines indicate the flux-density limits of the surveys, and the horizontal dotted lines show the range of radio luminosities that dominate the radio luminosity density of the universe (see text). The objects indicated for TOOT are a subset of the sample discussed here.

Fig. 1 shows the radio-luminosity $\left(\mathrm{L}_{151}\right)$ versus redshift diagram for various complete low-frequency RS redshift surveys, including TOOT. Selection at low frequency is essential in order to avoid orientation-dependent beaming effects that distort the source counts at high frequency. The 3CRR (1), 6CE (2), and 7CRS (3) surveys have a total of $356 \mathrm{RS}$, but still suffer from small number statistics and the fact that even the 7CRS does not reach the radio luminosity function (RLF) break at $\mathrm{z}>1$. As a result, there is still controversy about the form of the RLF evolution at high redshift, specifically whether there is a cut-off beyond $\mathrm{z}=2(4 ;$; $;$; 6 ). The TOOT survey on the other hand probes low-enough $\mathrm{L}_{151}$ at high redshift, and is sufficiently large, to resolve the form of the radio source population (RSP) evolution. Even at high redshift, TOOT contains objects with $\mathrm{L}_{151}$ typical of $3 \mathrm{CR}$ galaxies at $\mathrm{z} \sim 0.3$, allowing direct comparison of properties as a function of redshift without the usual problems of the redshift-luminosity degeneracy. The lower flux-density LBDS survey (7) misses the bulk of the luminosity density at all redshifts, and is dominated by very low $\mathrm{L}_{151} \mathrm{RS}$. The TOOT survey is the realisation of a survey proposed by Willott et al. (2001, W01 (6) ), and it has several key aims:

- Probe the existence of a redshift cutoff for the high-luminosity sources at $\mathrm{z}>2$. We will be able to finally get away from small number statistics and measure the magnitude of any high- $z$ space density decline.

- Establish whether the low-luminosity population evolves at all. At present 
Table 1

The Six TOOT Regions

\begin{tabular}{ccc|ccc}
\hline & $\mathrm{RA}(\mathrm{J} 2000)$ & $\operatorname{dec}(\mathrm{J} 2000)$ & & $\mathrm{RA}(\mathrm{J} 2000)$ & $\operatorname{dec}(\mathrm{J} 2000)$ \\
\hline TOOT00 & $00: 30$ & +35 & TOOT02 & $02: 00$ & +40 \\
TOOT08 & $08: 25$ & +27 & TOOT12 & $12: 50$ & +33 \\
TOOT16 & $16: 40$ & +45 & TOOT18 & $18: 00$ & +63 \\
\hline
\end{tabular}

we cannot discriminate reliably between constant space density and a rise by an order-of-magnitude: TOOT will establish the evolution to $\pm 0.1 \mathrm{dex}$.

- Probe large scale structure. Steep spectrum RS are ideal sparse tracers of LSS, having high bias and being easy to observe over large volumes (8; 9).

TOOT is the first survey to study the sources responsible for the bulk of the radio-luminosity density of the universe at high redshift (between the horizontal dotted lines in Fig. 1). They are typical in the same sense that $\mathrm{L}^{*}$ galaxies are typical of galaxies in the optical. Higher- $\mathrm{L}_{151} \mathrm{RS}$ are too rare to be important sources of energy input in the evolution of galaxies, as the RLF is steep. Radio source jet energy is coupled efficiently to the gas in the ISM and ICM and may account for $\sim 10 \%$ of the thermal energy of hot gas in clusters, a significant fraction of the total $(10)$. The injection of jet energy may also influence surrounding galaxies by heating the gas in the cluster environment. Study of the RS responsible for the radio luminosity density of the universe is hence of great interest.

At the TOOT flux-density limit, the RSP divides about equally into low- and high-luminosity sources. The low- $\mathrm{L}_{151} \mathrm{RS}$ (below $\log \left(\mathrm{L}_{151}\right)=26 \mathrm{WHz}^{-1} \mathrm{Sr}^{-1}$ in Fig. 1) do not show emission lines and have both FRI and FRII morphology, while the high- $\mathrm{L}_{151}$ ones have emission lines and FRII morphology. In TOOT we trace the low- $\mathrm{L}_{151}$ population to $\mathrm{z} \sim 2$. W01 have found a good fit to the RSP evolution for $\mathrm{S}_{151}>0.5$ Jy by combining a strongly evolving high- $\mathrm{L}_{151}$ population with a moderately evolving low- $\mathrm{L}_{151}$ population. TOOT can directly test the predictions of such models.

\section{Properties and Observational Status of TOOT}

TOOT targets six regions spread in RA in the northern hemisphere at declinations accessible to the Hobby-Eberly Telescope (HET), containing a total of $\sim 1000 \mathrm{RS}$ with $\mathrm{S}_{151}>0.1 \mathrm{Jy}$ (Table 1). We have high resolution radio maps of all sources, either from FIRST (11) or our own VLA observations. Most of the full sample has an identification, either from the Digital Sky Survey, R-band imaging from the McDonald Observatory $2.7 \mathrm{~m}$ Smith Reflector, or K-band imaging from UKIRT. 
Table 2

Current Status of the TOOT Survey Spectroscopy

\begin{tabular}{lcccl}
\hline Region & $\mathrm{S}_{151}(\mathrm{mJy})$ & area $_{\left(\text {sq. }^{\circ}\right)}$ & $\#$ spec & status \\
\hline TOOT00s & $>100$ & 4 & $\sim 50 / 54$ & $\sim 90 \%$ complete spectroscopy \\
TOOT08 & $>100$ & 20 & $\sim 140 / 153$ & $\sim 90 \%$ complete spectroscopy \\
TOOT12 & $>100$ & 25 & $\sim 260 / 265$ & $\sim$ complete spectroscopy \\
TOOT16_100 & $100-200$ & 63 & $\sim 40 / 121$ & $\sim$ complete IDs \\
TOOT18 & $>4000$ & 570 & $37 / 37$ & complete spectroscopy \\
\hline Totals & & & $\sim 440 / 472$ & TOOT00s, 08, 12 \\
& & & $\sim 520 / 630$ & full sample \\
\hline
\end{tabular}

We are currently about half way to our goal of measuring redshifts for the complete sample of $1000 \mathrm{RS}$. Table 2 summarizes the current completeness. Spectroscopy has been obtained with the HET Marcario Low Resolution Spectrograph ${ }^{1}$ (12), the ISIS spectrograph (13) on the WHT, or the IGI spectrograph (14) on the Smith Reflector. These facilities complement each other well: the ISIS has excellent blue response enabling the observation of Ly $\alpha$ emission for $z>1.6$ for the higher-luminosity sources that show emission lines; the HET has the aperture to measure absorption-line redshifts for the faint red galaxies with lower $\mathrm{L}_{151}$. For the high-redshift FRII population, spectra were often obtained blind, with the slit aligned to the radio axis.

As we have progressed, we have endeavoured to obtain completeness in subregions of the survey (TOOT00, 08, 12, and 18, see Table 2), and now have spectroscopy for half the sample. Obviously, a fraction of the redshifts will be unreliable or spurious - it is an unavoidable feature of such studies. The significantly smaller $6 \mathrm{C}$ and $7 \mathrm{CRS}$ surveys have achieved $>90 \%$ reliable redshifts. We estimate that we have reliable redshifts for $70-80 \%$ of the sample, with most of the unreliable/spurious ones associated with objects in the $0.6<\mathrm{z}<1.6$ redshift range, where most of the galaxies do not show emission lines, and are faint, resulting in poor $\mathrm{S} / \mathrm{N}$ ratio. As the HET continues to improve in performance, we will increase the completeness for this part of the sample, as large aperture is needed for these observations. At $\mathrm{z}>1.6$, most objects have sufficiently high $\mathrm{L}_{151}$ to show emission lines. Here the ambiguities center on objects with single-line redshifts, but K-band photometry ensures that alternative line IDs can usually be ruled out reliably. We are continuing to obtain K-band imaging to improve redshift estimates for the faintest objects.

1 The HET is operated by McDonald Observatory on behalf of The University of Texas at Austin, the Pennsylvania State University, Stanford University, LudwigMaximilians-Universität München, and Georg-August-Universität Göttingen. The Marcario LRS is a joint project of the HET partnership and the Instituto de Astronomía de la Universidad Nacional Autónoma de México. 


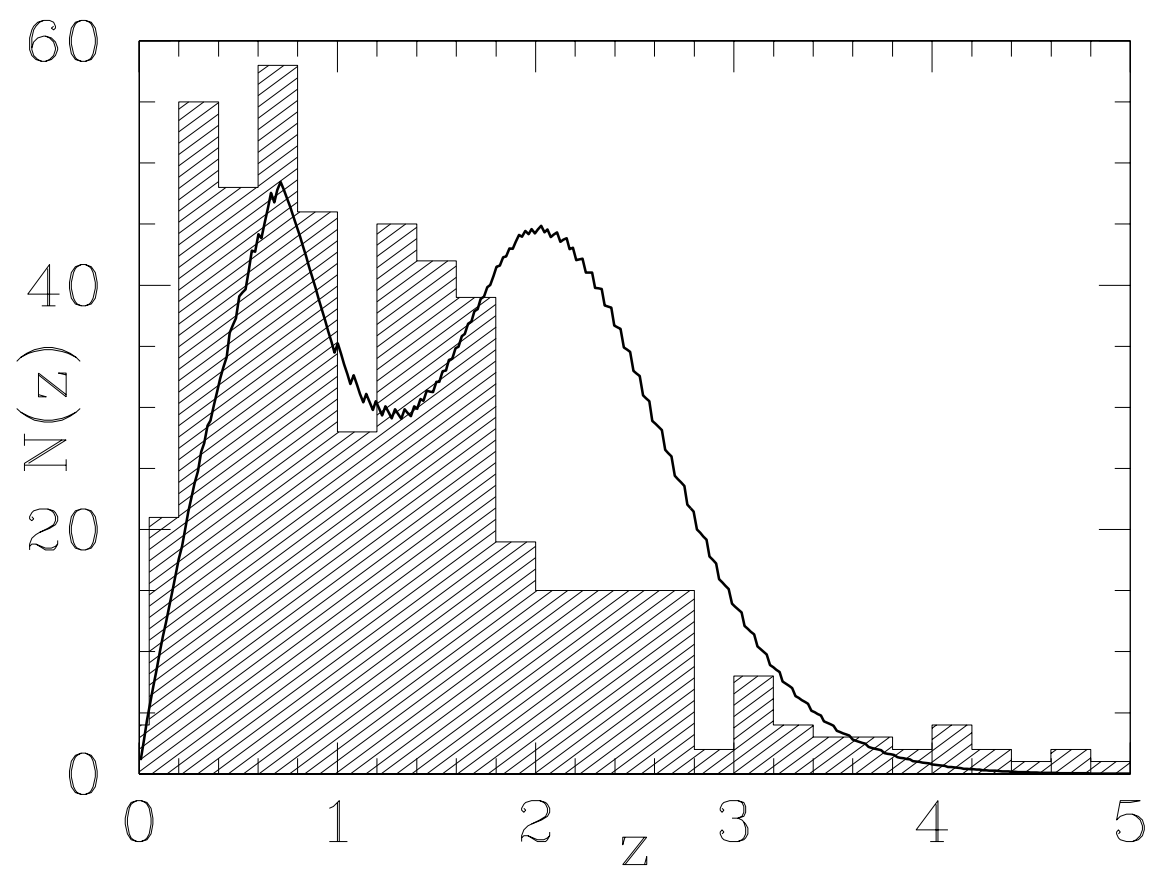

Fig. 2. Preliminary redshift distribution for half the TOOT survey. The solid curve is the W01 model C prediction, normalised appropriately (see text).

\section{The Preliminary Redshift Distribution}

Fig. 2 shows the redshift distribution for 442 sources in the complete TOOT00s, 08 , and 12 , fields. The $\sim 30$ sources without spectroscopy are not shown. The median redshift is $\mathrm{z}=1$. The $\mathrm{W} 01$ model $\mathrm{C}$, with an intermediate high-redshift drop-off, computed for $\Lambda=0.7, \Omega=0.3$ cosmology is overplotted at appropriate normalisation, for comparison. The double-peaked shape of the model prediction is due to the inflexion in the RLF between the two populations. The first conclusion from Fig. 2 is that the TOOT survey has been worthwhile: the model predictions are close to the observations for $\mathrm{z}<1$, but differ significantly at $z>2$. It is difficult to simply ascribe the disagreement to spurious/incomplete redshifts as there are too few objects without redshifts, and we do not expect spurious redshifts for $\mathrm{z}>1.6$, as long as the objects show emission lines. However, it is possible that the faint red radio galaxy population, for which redshifts will be most unreliable, contains examples of highly-reddened radio galaxies (EROs (15)) that should be at $\mathrm{z}>2$, but are erroneously ascribed lower redshifts. In order to fully account for the discrepancy, the EROs would be $20 \%$ of the sample. This is higher than the $8 \%$ found in the $7 \mathrm{CRS}$, for example (15), and it seems unlikely that we would have just missed those with $2<\mathrm{z}<3$. Indeed, the 7CRS EROs all lie between $1<\mathrm{z}<2$. On the other hand, our investigation of the properties of TOOT and 7CRS RS at $\mathrm{z} \sim 3(16)$ indicates that they are all reddened quasars, so a significant fraction of reddened, faint RS might be expected. With the current state of the spectroscopy we 
cannot rule out a significant population of EROs in TOOT at $\mathrm{z} \sim 2-3$, particularly as these objects have similar $\mathrm{L}_{151}$ to the lower-redshift $7 \mathrm{CRS}$ EROs. At higher redshift, the agreement with the W01 model $\mathrm{C}$ is quite good, indicating that any high-redshift cutoff is gradual. The factor of 10 increase in the space density of the low- $\mathrm{L}_{151}$ population to $\mathrm{z} \sim 1$ in the W01 models appears to give a good qualitative match to $\mathrm{N}(\mathrm{z})$ for $\mathrm{z}<1.5$. We will be comparing the results from TOOT to other models $(\underline{17})$ as we progress.

Acknowledgements We thank Julia Riley for obtaining the radio data on which TOOT is based. We thank Steve Croft, Kate Brand, Joe Tufts, Marcel Bergmann, and Pamela Gay for their efforts in obtaining and reducing the TOOT data. Chris Willott has had significant influence on the design of the TOOT survey. We also thank the staffs of the WHT, HET, UKIRT, and McDonald observatories, for their assistance in obtaining the dataset. This material is based in part upon work supported by the Texas Advanced Research Program under Grant No. 009658-0710-1999.

\section{References}

[1] R.A. Laing, J.M. Riley, M.S. Longair, MNRAS 204 (1983) 151

[2] S. Rawlings, S.A. Eales, M. Lacy, MNRAS 322 (2001) 523

[3] C.J. Willott, S. Rawlings, K.M. Blundell, M. Lacy, G.J. Hill, S.E. Scott, MNRAS 335 (2002) 1120

[4] J.S. Dunlop, J.A. Peacock, MNRAS 247 (1990) 19

[5] M.J. Jarvis, S. Rawlings, C.J. Willott, K.M. Blundell, S. Eales, M. Lacy, MNRAS 327 (2001) 907

[6] C.J. Willott, S. Rawlings, K.M. Blundell, M. Lacy, S.A. Eales, MNRAS 322 (2001) 536

[7] R.A. Windhorst, R.G. Kron, D.C. Koo, AA Suppl. 58 (1984) 39

[8] K. Brand, (2003) this conference

[9] G.J. Hill, New Era in Cosmology, T. Shanks, N. Metcalf, Eds., ASP Conf. Ser. 283 (2003) in press

[10] S. Rawlings, (2001) this conference

[11] R.H. Becker, R.L. White, D.J. Helfand, ApJ 450 (1995) 559

[12] G.J. Hill, H.E. Nicklas, P.J. MacQueen, C. Tejada, F.J. Cobos Duenas, W. Mitsch, Proc. SPIE 3355 (1998) 375

[13] http://www.ing.iac.es/PR/wht_info/whtisis.html

[14] G.J. Hill, M.J. Wolf, J.R. Tufts, E.C. Smith, Proc. SPIE 4842 (2003) in press

[15] C.J. Willott, S. Rawlings, K.M. Blundell, MNRAS 324 (2001) 1

[16] S. Rawlings, G.J. Hill, C.J. Willott, AJ (2003) in preparation

[17] J. Grimes, (2003) this conference 